\title{
Refraction of Spreading Induction in the Retina
}

\author{
By
}

\author{
Koiti Motokawa, Mitsuru Ebe, Mitsuo Komatsu \\ (本川 弘一) (江 部充) (小松三夫) \\ and Kei Watanabe \\ (渡 部 敬) \\ From the Physiological Laboratory, Tohoku University, Sendai
}

(Received for publication, March 28, 1958)

\section{INTRODUCTION}

In a previous paper by Motokawa and Komatsu'1 it was shown that the velocity of spreading induction depends on the state of adaptation; the velocity was found higher in a retinal area adapted to white light than in the dark-adapted one. In the present experiment colored light was used instead of white light, and it was found that the velocity was decreased below the value in the dark-adapted retina. From these findings it was inferred that the spreading induction would be refracted at the boundary between the dark-adapted and the light-adapted areas of the retina. The present paper deals with various aspects of refraction in the retina.

\section{EXPERIMENTAL}

\section{Method}

Spreading induction was investigated by our routine method, in which a pair of an inducer and a detector is used. The inducer, I, was a light stimulus for initiating spreading induction. The spreading induction caused by a blue inducer will be called "yellow spreading induction." The detector was a device for detecting arrival of spreading induction at any point at which the detector was placed. It consisted of a patch in hue complementary to the inducer and of a white test patch $1 \mathrm{~mm}$. in diameter. These stimuli were presented always at a distance of $30 \mathrm{~cm}$. in front of the eye of the subject.

The indirect induction established around a yellow detector was measured by the following procedure: The dark-adapted eye was exposed to the yellow patch of the detector for 2 seconds, and then to the white test patch, which was presented at a distance of $1 \mathrm{~mm}$. from the margin of the yellow patch. The interval separating the two photic stimuli has 
no influence on the result unless it is not longer than 20 seconds. The electrical excitability of the eye was measured 1.5 seconds after the end of the white test light. The value of electrical excitability was generally higher than that obtained following an isolate presentation of the white test light. The difference between the two values obtained with and without presenting the yellow patch represents the induction effect by the preceding yellow stimulus. The effect was expressed in per cent of the electrical excitability of the dark-adapted retina and designated "contrast effects (C.E.)."

The effect will not be found when the blue inducer was presented at some distance from the detector in the interval of the yellow and the white stimuli (the phenomenon of neutralization). When the spreading induction initiated by the blue inducer is prevented by any way from reaching the detector, the preestablished indirect induction at the detector will remain intact, and in consequence the contrast effect at the detector will be high. Thus the detector serves to detect arrival of spreading induction.

The details of arrangements of patches and experimental procedures will be described together with the results.

Results shape

Refraction of spreading induction by a light-adapted retinal area prismatic in

When the velocity of spreading induction through a prism-shaped retinal image is lower than in the surrounding dark areas, it is to be expected that the spreading induction will be refracted by the image towards the basis of the triangular image. On the contrary, it will be refracted towards the apex of the triangular image when the velocity through the image is higher than in the dark retinal area.

Experimental evidence was provided for the possibilities mentioned above in the following experiment: The arrangement of patches and the sequence of procedures are shown in the inset of Fig. 1. The apical angle of the prism-shaped patch was $15^{\circ}$. The patch was illuminated constantly by yellow light of 10 lux. The beam of spreading induction coming from the blue inducer, I, was refracted by the retinal image of the prism-shaped patch as indicated by a broken line in the inset of Fig. 1. The refracted beam was detected by the detector, $\mathrm{D}$, in the manner described above. The result obtained is shown by a continuous curve marked by solid triangles in Fig. 1. In this case neutralization occurred at a point $17^{\circ}$ from the middle point of the detector. The refraction of spreading induction is similar to that of light by a glass prism in this case. 


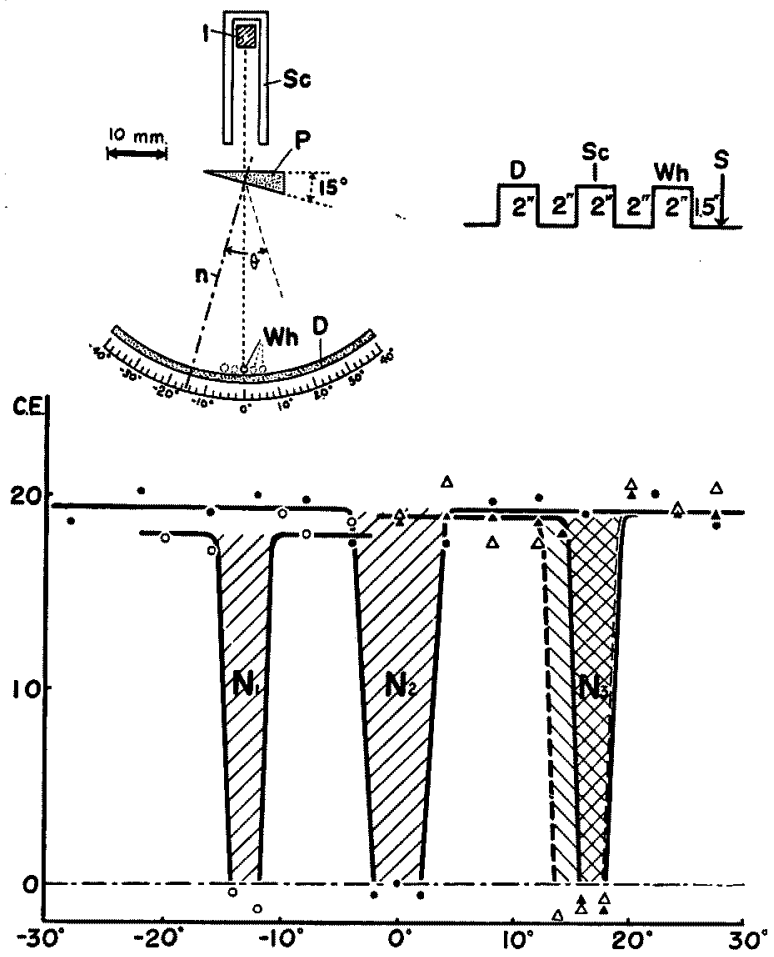

Fig. 1. Arrangement of patches and temporal sequence of experimental procedures for refraction of spreading induction. D : Yellow detector of 150 lux. I : Blue inducer of 150 lux. $\mathbf{n}$ : Normal. P : Prism-shaped patch of 10 lux with apical angle of $15^{\circ}$. S : Electric stimulus of 0.1 sec. Sc: White screen of 15 lux. Wh: White test light of 150 lux. Curve $N_{1}$ was obtained with $P$ illuminated by white light of 0.07 lux. Curve $N_{2}$ represents control obtained without any prism-shaped patch. Curve $\mathrm{N}_{8}$ was obtained with yellow prism-shaped patch illuminated continuously, and broken curve with the same patch illuminated intermittently.

In some other experiments the illumination was not so continuous as in the experiment mentioned above; the prism-shaped patch, $\mathrm{P}$, was presented together with the yellow detector, D, only for 2 seconds, so that the spreading induction caused by the subsequent inducer, I, traversed the after-image of the prism-shaped patch. The result obtained was qualitatively similar to that obtained in the experiments of constant exposure, as is shown by the broken curve marked by empty triangles in Fig. 1.

Next, white light of 0.07 lux was used instead of yellow light for illumination of the prism-shaped patch. Then refraction occurred towards the apex of the prism-shaped patch, neutralization being found at a point 
$13^{\circ}$ on the opposite side of the middle point of the detector (see the curve connecting open circles).

In the control experiement in which the prism-shaped patch was not presented, the other experimental conditions being the same as above, we found neutralization at the middle point of the detector (see the curve connecting solid circles).

Velocity and refraction of spreading induction as a function of intensities of adapting light

The velocity of spreading induction produced by a blue inducer, I, was measured with the arrangement shown in the inset of Fig. 2. An area, $\mathrm{M}$, which was adapted to yellow adapting light, had to be traversed

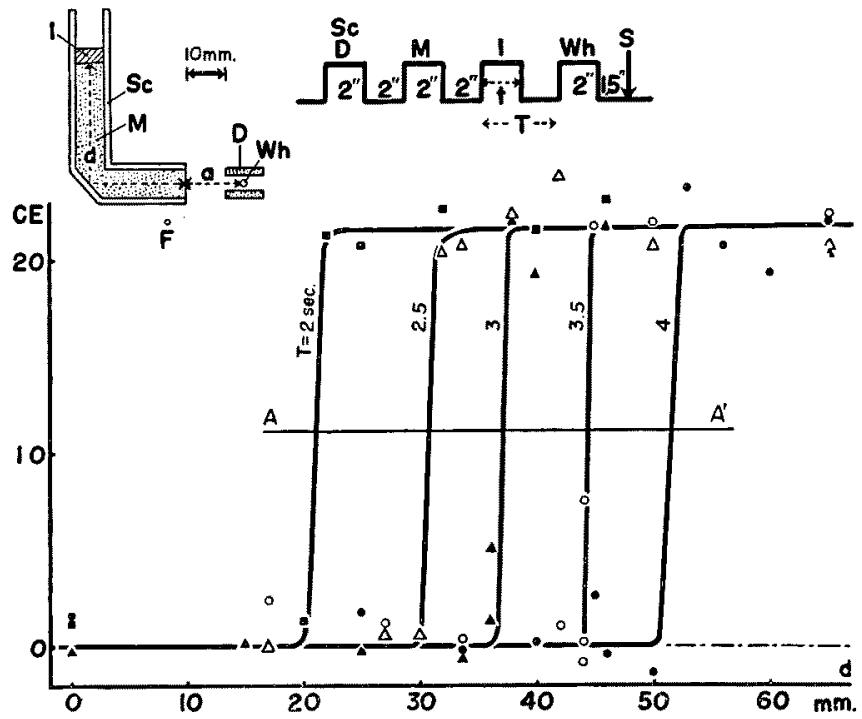

Fig. 2. Measurements of propagation velocity of spreading induction. Labeling of patches the same as in Fig. 1, except for $M$ which served for light adaptation of medium to be traversed by spreading induction. I : Blue inducer. D : Yellow detector. $M$ was illuminated by yellow light of 110 lux.

by the spreading induction initiated by the inducer, $\mathrm{I}$, before reaching the detector, $\mathrm{D}$. While the interval, $\mathrm{T}$, from the onset of $\mathrm{I}$ to that of the white test light, Wh, was fixed, the longitudinal distance, $\mathrm{d}$, of $\mathrm{M}$ was varied. In this experiment neutralization occurred when the distance, $\mathrm{d}$, was shorter than a certain value, but it failed to occur when the distance was too long. For example, neutralization occurred for $d$ shorter than $20 \mathrm{~mm}$. when $\mathrm{T}$ was fixed at 2 seconds. No neutralization occurred for longer distances, because the test of neutralization was made before the spreading induction reached the detector. The critical distance at which 


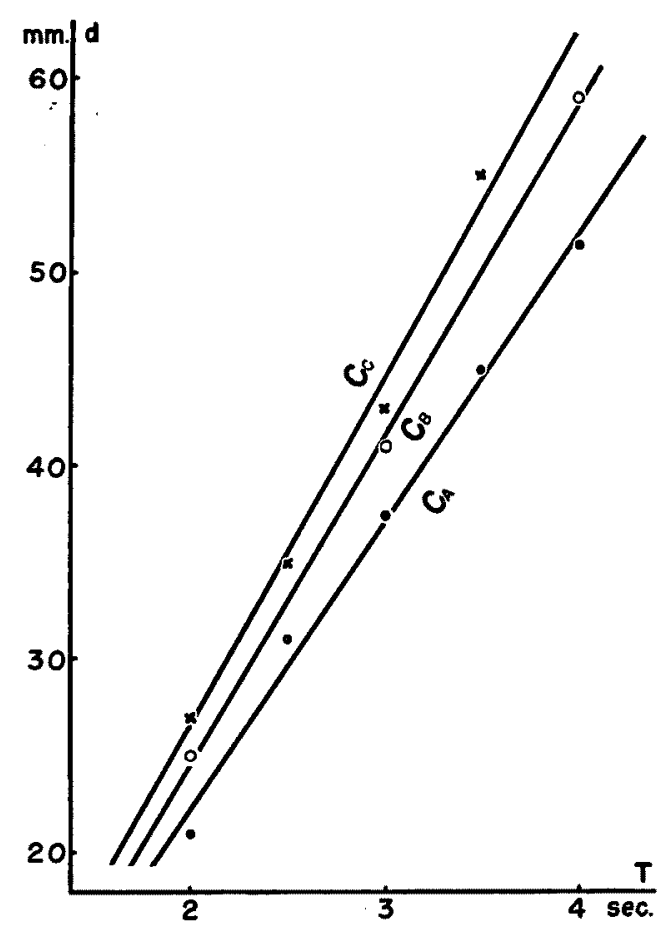

Fig. 3. Relation between conduction distance of spreading induction and conduction time. $C_{A}, C_{B}$ and $C_{C}$ refer to intensities of illumination for $M 110$, 1.1 and 0.07 lux respectively.

neutralization just failed became greater, as the time interval, $\mathrm{T}$, was lengthened. Such critical distances which are a function of interval, $\mathrm{T}$, will be called "propagation distances." In Fig. 3 propagation distances are plotted against time intervals, T. As can be seen from this figure, the relation is linear within the limits of experimental error, and this means that the velocity was uniform in the light-adapted area, M. The line labeled $\mathrm{C}_{\mathrm{A}}$ was obtained with yellow adapting light of 110 lux. Similar experiments were carried out at various adaptation levels of $M$. The lines $C_{B}$ and $C_{C}$ in Fig. 3 refer to adaptation levels at 1.1 lux and 0.07 lux respectively.

The slope of line seems to become steeper as the adaptation level is lowered. In other words, the higher the adaptation level, the slower the propagation velocity.

From the grounds to be stated later, the refractive index can be computed from the propagation velocities in the light-adapted and the darkadapted retinal areas. On the other hand we can determine the refractive index directly by measurements of refraction in the manner stated 
TAB LE I

Propagation Velocity of Yellow Spreading Induction in Retinal Area Adapted to Yellow Light

\begin{tabular}{c|c|c|c|c}
\hline \multirow{2}{*}{$\begin{array}{c}\text { Intensity of } \\
\text { yellow light } \\
\text { in lux }\end{array}$} & \multicolumn{2}{|c|}{$\begin{array}{c}\text { From experiments of propagation } \\
\text { velocity }\end{array}$} & \multicolumn{2}{c}{ From experiments of refraction } \\
\cline { 2 - 5 } & $\begin{array}{c}\text { Velocity in } \\
\text { retina (mm./sec.) } \\
\text { (observed) }\end{array}$ & $\begin{array}{c}\text { Refractive } \\
\text { index } \\
\text { (calculated) }\end{array}$ & $\begin{array}{c}\text { Velocity } \\
\text { (calculated) }\end{array}$ & $\begin{array}{c}\text { Refractive } \\
\text { index } \\
\text { (observed) }\end{array}$ \\
\hline 110 & 0.85 & 2.00 & - & - \\
10 & - & - & 0.83 & 2.02 \\
1.1 & 0.96 & 1.77 & - & - \\
0.12 & - & - & 0.94 & 1.81 \\
0.07 & 1.02 & 1.67 & - & - \\
0.05 & - & - & 1.08 & 1.57 \\
0.007 & 2.00 & 0.85 & - & -
\end{tabular}

above. Values of refractive index determined by these different methods are compared in Table I. It is to be noted that there is satisfactory agreement between them.

Refraction of spreading induction by a lens-shaped retinal image

A lens-shaped image was formed by a yellow patch of the same form, $\mathrm{L}$, to collect the spreading induction initiated by a blue inducer, I. A white screen, Sc, served to restrict the path of spreading induction to the lens-shaped image (see Fig. 4 A). The detector, D, used was a rectangular blue patch whose direct induction was to be neutralized by the spreading induction from I. A white test patch $2 \mathrm{~mm}$. in diamenter was presented to measure the direct induction caused by the blue patch of the detector. The detector was displaced from experiment to experiment so as to map the distribution of the neutralizing effect of the spreading induction from I. In Fig. $4 \mathrm{~A}$ the region at which the spreading induction arrived and neutralized the preestablished direct induction there is marked by open circles, and the surrounding areas in which no neutralization occurred by solid circles. In detail, the open circles represent values of C.E. between -2.0 and +3.0 , and the solid ones those between 13 and 16 . The shaded circles represent intermediate values, that is, those lying between 4.5 and 8.0. It is obvious from this finding that the path of spreading induction is converged by the lens-shaped retinal image in a manner very similar to the action of a convex lens. By a physical convex lens light from a point source is focused on a point where the density of light is higher than at any point on the light path.

The above-mentioned experiment can, however, tell nothing about the density of spreading induction, because neutralization occurs in the 


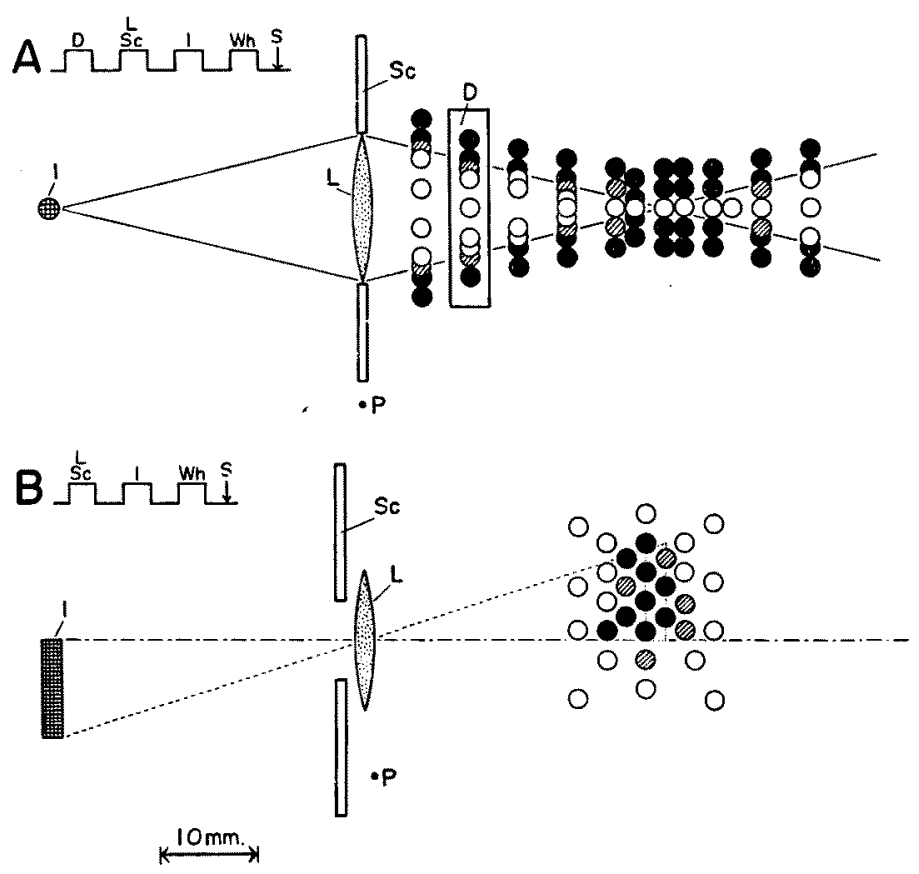

Fig. 4. Convergence of spreading induction by a lens-shaped retinal image. I : Blue inducer. Sc: White screen. L: Yellow lens-shaped patch. D : Blue detector. Upper diagram A represents distribution of neutralizing effect of spreading induction from I upon direct induction caused by $\mathrm{D}$ which was placed at various places in field so as to map distribution of neutralization. Occurrence of neutralization is indicated by open circles, and its absence by solid circles. Lower diagram $B$ represents distribution of contrast effects caused by spreading induction collected by $\mathrm{L}$. Contrast effects fully developed are represented by solid circles, and its absence by open circles, shaded ones representing intermediate effects.

manner of all-or-none. If spreading induction is focused on a certain restricted part of the retina an induction effect will become manifest in this part. Based upon this supposition, it was attempted in the following experiment to measure the induction effect which would be caused by spreading induction concentrated by the lens-effect. The procedure used was the same as that employed for measuring the indirect induction around a retinal image: As the inducer was blue in hue the indirect induction caused by it can be measured by the difference of electrical excitabilities at $3 \mathrm{sec}$. after the sequence of stimuli, blue - white and at $3 \mathrm{sec}$. after the white stimulus alone. The result obtained is shown in Fig. 4 B. In this figure values of C.E. between -2.0 and +2.0 are expressed by open circles. These low values of C.E. indicate that the density of spreading 
induction was nearly zero at these points. In a certain restricted region of the retina values of G.E. were as high as from 8.5 to 14.5 , as indicated by solid circles. Shaded circles in the figure represent intermediate values of C.E. It is to be noted that the region of high C.E. values just corresponds with the geometrical image of $I$ formed by the lens-action of $L$. In control experiments in which no lens-shaped patch was presented, values of C.E. were found always zero within the limits of experimental error everywhere on the right side of the screen, Sc.

Refraction of yellow spreading induction by a prism-shaped retinal area adapted to spectral lights of various wave-lengths

In most experiments stated above the prism-shaped patch was illuminated by yellow light, and the refraction of yellow spreading induction by such a yellow prism-shaped image was investigated. In the following experiments spectral lights of various wave-lengths were used for illumination of the prism-shaped patch, the spreading induction being the same as above.

The refractive index was measured after the method of the least deviation angle which is used for determination of the refractive index of a prism in physics. The arrangement of patches and the temporal sequence of procedures are shown in the insets of Fig. 5. The set of patches,

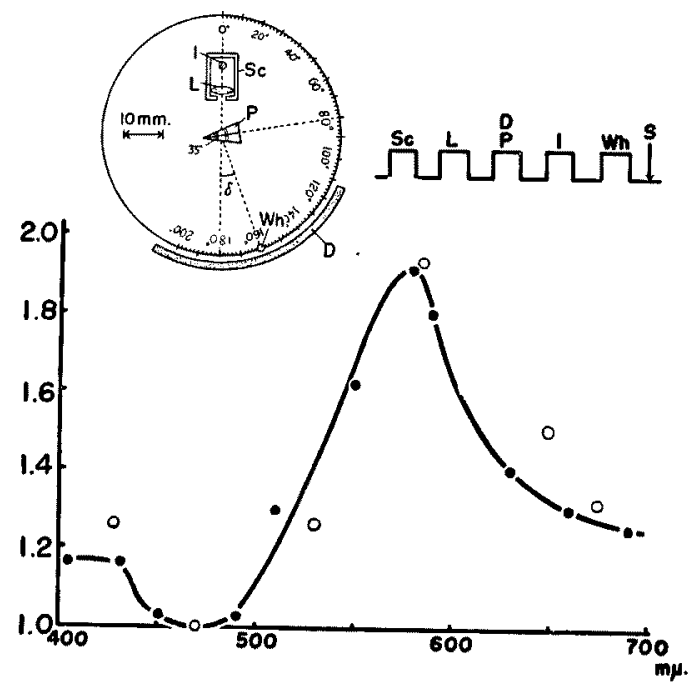

Fig. 5. Measurements of refractive index. Prism-shaped patch, $P$, was illuminated by spectral lights of various wave-lengths. Set of patches I, Sc and $\mathrm{L}$ represents a collimator. Least deviation angle was determined by procedures described in text. Curve marked by solid circles was obtained with refraction by after-image of $\mathrm{P}$, and that marked by open circles with refraction by $\mathrm{P}$ during illumination. 
I, $\mathrm{L}$ and Sc, served to produce a beam of yellow spreading induction. It was refracted by the prism-shaped after-image of patch, $\mathrm{P}$, and reached a certain part of the detector, D. P could be rotated around a point, and the angle of rotation could be read. The prism-shaped patch was placed in such a way that the condition of the least deviation angle was satisfied. The procedures actually used will be explained by an example. In the example to be stated the prism-shaped patch was illuminated with yellow light of $589 \mathrm{~m} \mu$. The patch was so placed that the bisecting line of the apical angle pointed to $71^{\circ}$ on the scale. The part around $71^{\circ} \times 2$ of the detector was searched for neutralization. By the method of trial and error we found that for a combination of $74^{\circ}$ of the bisecting line and $148^{\circ}$ of the detector a complete neutralization occurred. In this case the least deviation angle was given by the difference between $180^{\circ}$ and $148^{\circ}$.

If the apical angle and the least deviation angle are denoted by $\theta$ and $\delta$ respectively, the refractive index, $\mathrm{n}$, will be given by the following formula :

$$
\mathbf{n}=\frac{\sin \frac{1}{2}(\theta+\delta)}{\sin \frac{\theta}{2}}
$$

Putting $35^{\circ}$ for $\theta$ and $32^{\circ}$ for $\delta$ in the formula, we obtained 1.82 as the refractive index of the yellow prism-shaped image for the yellow spreading induction.

Similar measurements were carried out with spectral lights of various wave-lengths for illumination of the prism-shaped patch. The results obtained are shown in Table II, and their graphical representation is found in Fig. 5. As can be seen in this figure, the refractive index determined with the yellow spreading induction is highest for a retinal area adapted to yellow light and lowest for an area adapted to blue light.

As the method used above was too complicated, some experiments were performed with the simpler method described in Fig. 1. The illumination of the prism-shaped patch was continuous in these experiments. In spite of these differences in method and condition the results obtained were very similar to those obtained by the complicated method (cf. the curve marked by open circles with that marked by solid ones). It is also to be noted that these two kinds of experiments were carried out by different experimenters on different subjects, and that the dates of the experiments were separated by 6 years.

For comparison some experiments were performed on the propagation velocity, in order to study the dependence of velocity on the wavelength of adapting light for the medium to be traversed by yellow spreading induction. The method used was the same as described in Fig. 2. The 
TABLE II

Agreement of Refractive Indices Computed from Propagation Velocities with Those Determined by Experiments of Refraction

\begin{tabular}{c|c|c|c}
\hline $\begin{array}{c}\text { Wave-length of } \\
\text { adapting light } \\
(\mathrm{m} \mu)\end{array}$ & $\begin{array}{c}\text { Velocity in } \\
\text { retina } \\
(\mathrm{mm} . / \mathrm{sec} .)\end{array}$ & \multicolumn{2}{|c}{ Refractive index } \\
\cline { 2 - 3 } 690 & 1.36 & Computed & Observed \\
660 & 1.30 & 1.25 & 1.24 \\
630 & 1.22 & 1.30 & 1.30 \\
589 & 0.94 & 1.40 & 1.40 \\
580 & - & 1.82 & 1.81 \\
550 & 1.05 & - & 1.95 \\
510 & 1.30 & 1.62 & 1.62 \\
490 & - & 1.30 & 1.30 \\
450 & 1.67 & - & 1.03 \\
430 & 1.56 & 1.02 & 1.03 \\
405 & 1.50 & 1.09 & 1.14 \\
& & 1.13 & 1.14
\end{tabular}

results obtained are compared with those obtained from the experiments of refraction in Table II.

\section{Discussion}

In general, refraction occurs when the conduction velocity in one medium is different from that in another in contact with the former. The refractive index is given by the ratio of the conduction velocities in the two media.

The velocity of spreading induction was determined with three different methods ${ }^{2,3), 4)}$ with consistent results. It is about $1.7 \mathrm{~mm}$. per second in the dark-adapted retina. As has been shown above, the velocity of yellow spreading induction is lower in the retina adapted to colored light than in the dark-adapted retina. In consequence the refractive index is higher than unity.

On the contrary, the velocity in the retina adapted to white light is higher than that in the dark-adapted retina so that the refractive index is lower than unity. We do not know as yet the reasons why the velocity depends so on the adaptation state of the retina. The mechanism will remain obscure till we get more information about the properties of spreading induction. As has been shown in Tables I and II, we see satisfactory agreement between refractive indices determined by the measurements of refraction and those computed from propagation velocities. This finding together with the facts of reflection of spreading induction ${ }^{1,4}$ ) 
seems to suggest the wave-nature of spreading induction. Experimental evidence will be provided by further experiments on diffraction of spreading induction.

We have seen above that an induction effect occurred in a certain restricted retinal area when spreading induction was focused by the action of a lens-shaped patch. One may ask whether the induction effect is made visible. We also tried to make it visible, but have not yet succeeded in such attempts. The indirect induction around a retinal image is visible as the phenomenon of contrast. In such cases values of C.E. are generally greater than 20 in the region in which simultaneous contrast is observable. With the induction effect caused by the lens-action values of G.E. were 16 at the highest, and such low values of C.E. may not be sufficient to make the effect visible.

\section{SUMMARY}

It was shown by the method of electrostimulation of the eye that spreading induction was refracted when a prism-shaped retinal image was formed on the way of its propagation through the retina.

1. The degree of refraction depended on the intensity and also the wave-length of light illuminating a prism-shaped patch whose image was formed on the retina.

2. The propagation velocity of spreading induction was measured to investigate its dependence on the intensity and the wave-length of adapting light. In the retina adapted to white light the velocity was higher than in the dark-adapted retina, while in the retina adapted to colored light the velocity was found lower than in the dark-adapted retina.

3. The refractive index computed from propagation velocities was in satisfactory agreement with that determined by measurements of refraction.

4. It was shown by many examples that the law of geometrical optics holds for spreading induction in the human retina.

\section{References}

1) Motokawa, K. \& Komatsu, M., Tohoku J. Exp. Med., 1958, 67, 149.

2) Motokawa, K., Iwama, K. \& Ebe, M., ibid., 1954, 59, 11.

3) Katayama, S. \& Aizawa, T., ibid., 1956, 64, 179.

4) Motokawa, K. \& Komatsu, M., ibid., 1957, 67, 29. 